\title{
One-Year Course of Periprocedural Anticoagulation in Atrial Fibrillation Ablation: Results of a German Nationwide Survey
}

\author{
Alexandru Gabriel Bejinariu ${ }^{a} \quad$ Hisaki Makimoto ${ }^{a}$ Reza Wakilib Shibu Mathew ${ }^{c}$ \\ Jedrzej Kosiuk ${ }^{d}$ Dominik Linz ${ }^{\text {e, }}$, g Johannes Steinfurt ${ }^{\text {h }}$ Dirk G. Dechering ${ }^{i}$ \\ Christian Meyer ${ }^{j} \quad$ Christian Veltmann ${ }^{k} \quad$ Malte Kelm $^{\mathrm{a}, l} \quad$ Gerrit Frommeyer $^{\mathrm{i}}$ \\ Lars Eckardt ${ }^{\mathrm{i}}$ Thomas Deneke ${ }^{\mathrm{m}}$ David Duncker ${ }^{k}$ Patrick Müller ${ }^{\mathrm{a}, \mathrm{i}}$
}

${ }^{a}$ Cardiac Arrhythmia Service, Division of Cardiology, Pulmonology and Vascular Medicine, Faculty of Medicine, Heinrich-Heine University, Düsseldorf, Germany; ${ }^{b}$ Department of Cardiology and Vascular Medicine, West German Heart and Vascular Center Essen, University Duisburg-Essen, Duisburg-Essen, Germany; ' ${ }^{\mathrm{D}}$ Department of Cardiology, Asklepios Klinik St. Georg, Hamburg,

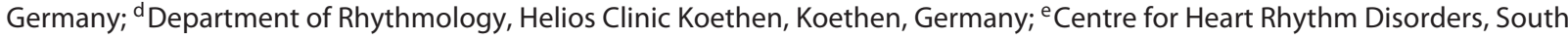
Australian Health and Medical Research Institute (SAHMRI), University of Adelaide and Royal Adelaide Hospital, Adelaide, SA, Australia; fDepartment of Cardiology, Maastricht University Medical Centre, Maastricht, The Netherlands; ${ }^{9}$ Department

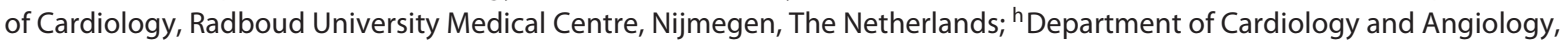
Heart Center Freiburg University, Freiburg, Germany; 'Department of Cardiology II - Electrophysiology, University Hospital of Münster, Münster, Germany; 'Department of Cardiology-Electrophysiology, University Hospital Hamburg, University Heart Center, Hamburg, Germany; ${ }^{k}$ Rhythmology and Electrophysiology, Cardiology and Angiology, Hannover Medical School, Hannover, Germany; 'CARID, Cardiovascular Research Institute Düsseldorf, Faculty of Medicine, Heinrich-Heine-University, Düsseldorf, Germany; ${ }^{\mathrm{m}}$ Heart Center Bad Neustadt, Clinic for Interventional Electrophysiology, Bad Neustadt, Germany

\section{Keywords}

Atrial fibrillation ablation · Oral anticoagulation ·

Periprocedural strategy · Survey

\begin{abstract}
Introduction: Periprocedural oral anticoagulation (OAC) strategies for atrial fibrillation (AF) ablation procedures are changing rapidly. Objective: To assess the management and course of periprocedural OAC for AF ablation procedures in experienced electrophysiology (EP) centers in Germany over the last 12 months. Methods: The data are based on an electronic questionnaire, which was sent to 35 experienced EP centers in September 2018 and then exactly 1 year later. Participants provided information on their periprocedural OAC management, the handling with dual therapy (OAC plus single antiplatelet therapy), the availability of specific anti-
\end{abstract}

karger@karger.com www.karger.com/crd

Karger $\frac{1}{\%}$

BOPEN ACCESS
(C) 2020 The Author(s)

Published by S. Karger AG, Basel

This article is licensed under the Creative Commons AttributionNonCommercial-NoDerivatives 4.0 International License (CC BYNC-ND) (http://www.karger.com/Services/OpenAccessLicense). Usage and distribution for commercial purposes as well as any distribution of modified material requires written permission. dotes, the transseptal puncture approach, and noteworthy complications. Results: Responses were received from all 35 centers and represent 10,010 AF ablation procedures annually. In 2018, the administration of vitamin $\mathrm{K}$ antagonist (VKA) was continued throughout the procedure at all centers $(100 \%)$. In contrast, the majority of centers used minimally interrupted periprocedural non-vitamin $\mathrm{K}$ antagonist oral anticoagulants (NOAC) (54.3\%), 13 centers (37.2\%) completely interrupted NOAC, and only 3 centers (8.5\%) continued NOAC throughout the procedure. At the 1-year followup survey, 32 centers were found to have continued their previous strategy of periprocedural $O A C$ and 3 changed from a minimally interrupted to a continued NOAC strategy. Of note, 30 centers $(85.7 \%)$ performed transseptal puncture

Patrick Müller and David Duncker contributed equally to this work and share last authorship. 
fluoroscopically without additional cardiac imaging. In the setting of uninterrupted periprocedural OAC management, no relevant complications were noted. Conclusion: Our survey shows marked heterogeneous periprocedural OAC management at experienced EP centers in Germany. Whereas continuation of VKA has already been integrated into clinical practice, the majority of centers still use a minimally interrupted NOAC strategy.

() 2020 The Author(s)

Published by S. Karger AG, Basel

\section{Introduction}

Catheter ablation of atrial fibrillation (AF) has become the standard therapy for patients with drug-refractory symptomatic AF [1]. Balancing both procedural thromboembolic and bleeding risks, anticoagulation strategies before, during, and after AF ablation are of major clinical relevance. Numerous studies investigating continuous periprocedural oral anticoagulation (OAC) with vitamin $\mathrm{K}$ antagonists (VKA) have already shown a reduction in both embolic and bleeding risks when compared to bridging with low-molecular-weight heparin (LMWH) $[2,3]$. There is a rapidly growing body of evidence suggesting that the uninterrupted administration of non-vitamin $\mathrm{K}$ antagonist oral anticoagulants (NOAC) during AF ablation is noninferior to continuation of VKA with a therapeutic international normalized ratio (INR) target of 2-3 [4-7]. As a consequence, recent consensus documents have recommended continuation (truly uninterrupted), but also allow holding 1-2 doses of NOAC prior to the procedure (minimally interrupted), especially if transseptal puncture is performed without additional cardiac imaging $[8,9]$. However, evidence of larger controlled trials regarding the minimally interrupted strategy are lacking.

The aim of this nationwide survey was to provide an insight into the current practice of periprocedural OAC in $\mathrm{AF}$ ablation procedures over the last 12 months at experienced electrophysiology (EP) centers in Germany.

\section{Methods}

The survey was conducted at 35 certified EP centers in Germany with the approval and support of the national working group on arrhythmias of the German Cardiac Society (DGK). An electronic questionnaire consisting of 14 questions was sent via e-mail in September 2018 using the surveymonkey.com platform (SVMK Inc., San Mateo, CA, USA). A follow-up survey consisting of 4 questions was sent after exactly 1 year to all the participating centers, i.e., in September 2019.

Periprocedural Anticoagulation in AF Ablation

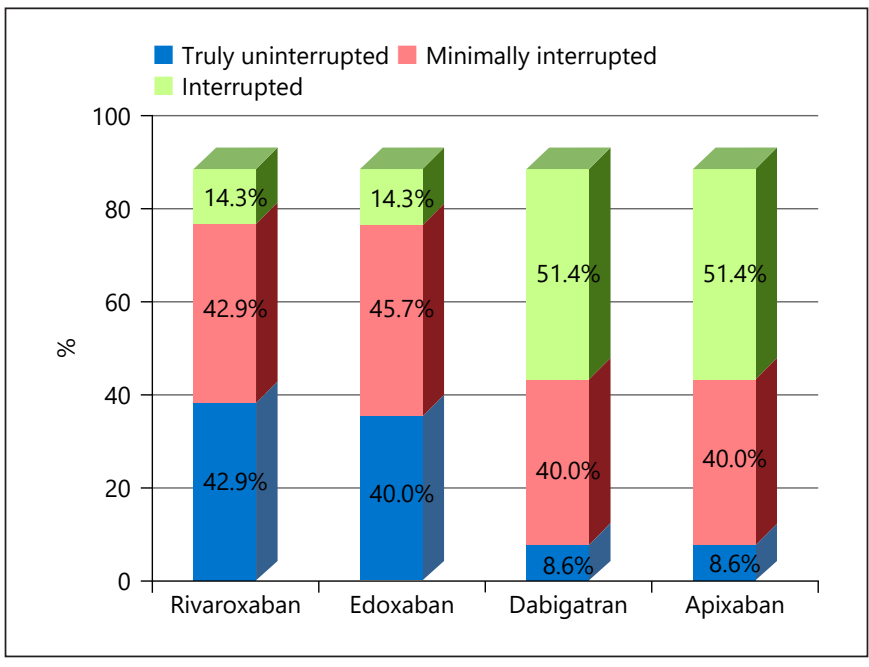

Fig. 1. Periprocedural strategies for specific NOAC.

The following aspects were covered in the baseline survey: the type of hospital; the annual AF ablation volume; peri- and intraprocedural anticoagulation regimes with all approved NOAC (rivaroxaban, edoxaban, apixaban, and dabigatran) in patients without indications for a reduced dose, VKA, and bridging with LMWH, respectively; the use of antidotes (prothrombin complex concentrate [PPSB], idarucizumab, and protamine); AF ablation under dual therapy (single antiplatelet and anticoagulation agent); and noticeable complications. The 1-year follow-up survey focused on the following aspects: any change in periprocedural anticoagulation strategy, the use of a transseptal puncture approach, and noticeable complications. The detailed questionnaires can be found in the online supplementary material (for all online suppl. material, see www.karger.com/doi/10.1159/000509399).

An uninterrupted VKA strategy was defined as maintaining VKA with a therapeutic INR of $2-3$.

The periprocedural NOAC strategies were defined as follows:

1 A truly uninterrupted strategy, i.e., if the patient received the standard NOAC dose before or after AF ablation on the day of the procedure for once-daily NOACs, or the standard NOAC dose before and after AF ablation on the day of the procedure for twice-daily NOACs.

2 A minimally interrupted strategy, i.e., if the NOAC dose was reduced or skipped on the day of the procedure for once-daily NOACs, or if the NOAC dose was skipped only before the procedure on the day of ablation for twice-daily NOACs.

Descriptive statistics were performed with SPSS v23 (SPSS Inc., Chicago, IL, USA).

\section{Results}

All 35 EP centers responded to the survey and answered all questions. In total, the survey reflects 10,010 AF ablation procedures per year in Germany. Annually, 


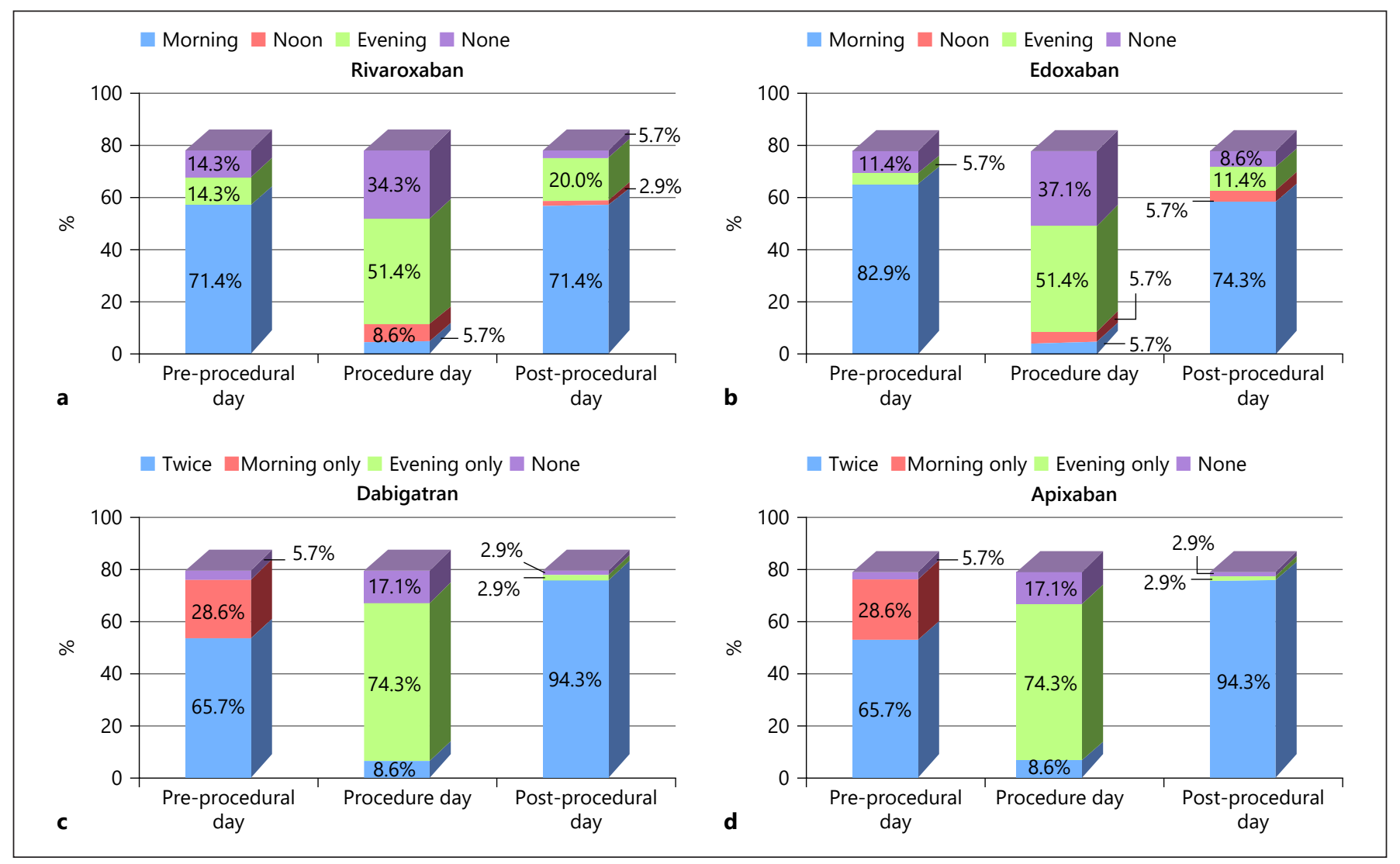

Fig. 2. Day-specific use of NOAC. a Rivaroxaban. b Edoxaban. c Dabigatran. d Apixaban.

the vast majority of EP centers $(n=20,57 \%)$ were found to perform $>200 \mathrm{AF}$ ablation procedures, 6 centers $(17 \%)$ performed $151-200$ procedures, 6 (17\%) performed $101-$ 150 procedures, and $3(9 \%)$ performed $75-100$ procedures.

\section{Baseline and Follow-Up Periprocedural Handling of \\ $O A C$}

Patients anticoagulated with VKA underwent AF ablation without bridging at all centers. A truly uninterrupted periprocedural NOAC strategy (for all NOACs) was performed at 3 centers $(8.6 \%)$, a minimally interrupted strategy at 19 centers $(54.3 \%)$, and an interrupted at 13 centers (37.1\%). The periprocedural strategies for specific NOAC are summarized in Figure 1 and the detailed day-specific use of NOAC is shown in Figure 2a-d. Bridging with heparin was used at 5 centers (14.3\%) if the NOAC dose was paused in the evening on the day of the procedure, as follows: LMWH at 3 centers and unfractioned heparin at 2 centers, with a target activated partial thromboplastin time (aPTT) of $60-80 \mathrm{~s}$.
In the 1-year follow-up survey, $3 \mathrm{EP}$ centers indicated they had changed the periprocedural NOAC strategy from a minimally interrupted to an uninterrupted strategy; the remaining 13 centers maintained an interrupted NOAC strategy (Fig. 3).

\section{Handling of Additional Antiplatelet Therapy}

Nineteen centers (54.3\%) reported AF ablation procedures under dual therapy (OAC plus aspirin or clopidogrel), 6 centers (17.1\%) favored early discontinuation of platelet inhibition according to current guidelines, and 10 centers $(28.6 \%)$ postponed the procedure until the end of the required time of dual therapy.

\section{Transseptal Puncture Approach}

The majority of EP centers (85.7\%) performed fluoroscopically guided transseptal puncture without additional intraprocedural cardiac imaging. Four centers (11.4\%) used guided transseptal puncture and transesophageal echocardiography and 1 center (2.9\%) used additional intracardiac echocardiography. 


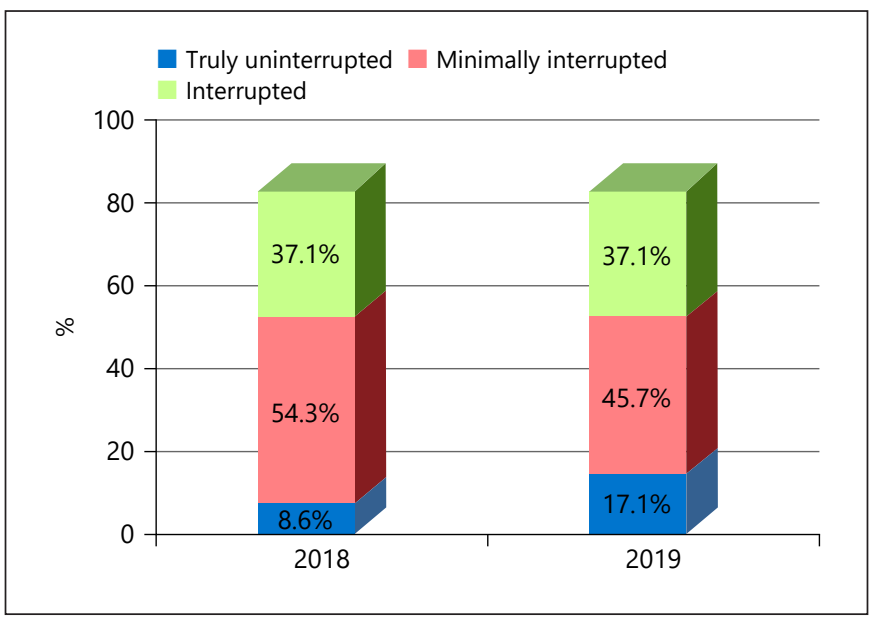

Fig. 3. One-year course of periprocedural NOAC strategies.

\section{Administration of Antidotes}

Administration of protamine after AF ablation was used routinely at 12 centers (34.3\%) and in cases with bleeding complications at 11 centers (31.4\%); 12 centers (34.3\%) did not use protamine at all. Regarding the management of periprocedural bleeding complications, 16 centers $(45.7 \%)$ had PPSB and idarucizumab at their disposal, 9 centers $(25.7 \%)$ had PPSB only, and 10 centers (28.6\%) did not have any substances readily available (2 of these centers had already used idarucizumab for acute bleeding complications in the EP laboratory).

\section{Reasons for Not Using an Uninterrupted \\ Periprocedural DOAC Strategy}

The 29 centers which did not adopt a truly uninterrupted periprocedural DOAC strategy stated the following main reasons for this approach: (1) the use of transseptal puncture without additional cardiac imaging, (2) the fear of major bleeding, and (3) the absence of antidotes. Only 2 centers (5.7\%) reported fewer bleeding complications when using an uninterrupted periprocedural OAC strategy instead of the previous heparinbridging therapy.

\section{Discussion}

This nationwide survey provides an insight into the current periprocedural OAC management of patients undergoing AF ablation in Germany. The survey was performed 5 and 17 months after the release of "The 2018 European Heart Rhythm Association (EHRA) Practical
Guide on the Use of Non-Vitamin K Antagonist Oral Anticoagulants in Patients with Atrial Fibrillation" [9]. This practical guide recommends using a truly uninterrupted strategy, if transseptal puncture is performed with periprocedural imaging (i.e., intracardiac echocardiography). Otherwise, it is recommended that the last dose of NOAC be administered $12 \mathrm{~h}$ before the procedure (i.e., the minimally interrupted strategy).

The main findings of our survey are:

1 The periprocedural uninterrupted VKA strategy is implemented in clinical practice in Germany.

2 The periprocedural NOAC strategy differs significantly among EP centers.

3 The majority of EP centers in Germany favor a minimally interrupted periprocedural NOAC strategy.

4 The periprocedural NOAC strategy remained almost unchanged over a period of 12 months.

As AF ablation is an elective procedure, efficacy and safety are both of significant clinical importance. The safety aspect mainly concerns periprocedural complications, primarily due to stroke and bleeding events.

The periprocedural continuation of OAC in patients undergoing AF ablation was tested in the COMPARE trial [3], which involved 1,584 patients undergoing AF ablation being randomized to continuation of VKA versus bridging with LMWH. The primary end point was thromboembolic events during the $48 \mathrm{~h}$ after the index procedure. The authors showed that continuation of VKA was superior to the interruption of VKA and bridging with LMWH.

The VENTURE-AF trial [4] and the RE-CIRCUIT trial [5] compared major bleeding as the primary end point in patients undergoing AF ablation on uninterrupted rivaroxaban or dabigatran, respectively, to uninterrupted VKA. In detail, the VENTURE-AF trial showed similar bleeding event rates, but the RE-CIRCUIT trial demonstrated significant lower bleeding complications under dabigatran. The AXAFA-AFNET 5 [6] and ELIMINATEAF [7] trials were designed with a primary composite end point of all-cause death, stroke, or major bleeding events. Both trials showed noninferiority of uninterrupted apixaban or edoxaban, respectively, versus uninterrupted VKA. Recently, Romero et al. [10] published a meta-analysis of randomized controlled trials comparing uninterrupted NOACs and uninterrupted VKA during AF ablation. This meta-analysis of $1,716 \mathrm{AF}$ patients confirmed the previous data and showed no significant differences in terms of major bleeding events, thromboembolic complications, or silent strokes on brain magnetic resonance imaging. 
Despite the abovementioned randomized clinical trials supporting the truly uninterrupted periprocedural approach and recommendation of a truly uninterrupted strategy in the EHRA Practical Guide [9], our nationwide survey demonstrated that only a minority of experienced EP centers in Germany have implemented a truly uninterrupted periprocedural NOAC strategy into clinical practice. Instead, most centers apply a minimally interrupted NOAC strategy. When considering the 2 types of NOAC administration (rivaroxaban and edoxaban vs. apixaban and dabigatran) dosed once and twice daily, there was greater adherence to the truly uninterrupted approach with the once-daily dose.

Interestingly, more than one-third of the EP centers (37\%) chose an interrupted strategy. A possible explanation for this phenomenon may be the relatively small amount of time between the publication of the abovementioned guideline and when we conducted the survey.

Focused on a minimally interrupted approach, the AEIOU [11] and ABRIDGE-J [12] randomized trials compared the use of minimally interrupted apixaban or dabigatran to uninterrupted use of VKA in patients undergoing AF ablation. In detail, the AEIOU trial showed no differences in major bleeding and a low rate of thromboembolic events in both study arms [11] whereas the ABRIDGE-J trial indicated lower bleeding events with minimally interrupted use of dabigatran [12]. Nakamura et al. [13] and Nagao et al. [14] compared the use of minimally interrupted NOAC versus uninterrupted NOAC use in patients undergoing AF ablation. Nakamura et al. [13] showed no significant differences regarding the primary end points of symptomatic thromboembolism and major bleeding, Nagao et al. [14] revealed a higher incidence of silent strokes in the minimally interrupted NOAC group. However, considering the small sample size and the low incidence of thromboembolic events, neither the AEIOU nor the ABRIDGE-J trial was powered to establish the superiority of the minimally interrupted NOAC strategy.

Based on available evidence from randomized trials, recent consensus documents have recommended using an uninterrupted NOAC strategy. Although the consensus documents also allow a minimally interrupted NOAC strategy, there is little evidence to support this approach $[8,9]$.

In our survey, we found that transseptal puncture in Germany is predominantly performed without additional cardiac imaging, and specific antidotes are not yet widely available. These factors may explain why the majority of EP centers in Germany prefer a minimally interrupted NOAC strategy for patients undergoing AF abla- tion. However, data on the performance of this strategy, specifically in patients for whom transseptal puncture is performed without additional cardiac imaging, are not available. To fill this gap in the evidence, larger studies investigating the safety and efficacy of minimally interrupted NOAC therapy, that include patients for whom transseptal puncture is predominantly performed without cardiac imaging, are needed to produce definite evidence-based recommendations in the future.

Regarding the differences in number of procedures performed under VKA versus NOAC, we expect that, for $>60 \%$ of procedures, a NOAC was the preferred substance, considering the prescription number of oral anticoagulants in Germany [15].

In addition to differences in periprocedural OAC regimes, this survey shows that more than half of the interviewed $\mathrm{EP}$ centers performed $\mathrm{AF}$ ablation under dual therapy (OAC and platelet inhibitors), as safety data on this topic are missing. Besides, only a third of the centers used protamine routinely at the end of the procedure.

The survey reflects a relevant proportion of AF ablation procedures performed at experienced EP centers in Germany, covering $>10,000$ AF ablations per year. Nevertheless, this study has some limitations. The invitation process was limited to $35 \mathrm{EP}$ centers in Germany, creating a selection bias. Furthermore, possible complications may have been underreported, so we were not able to analyze the complication rates associated with periprocedural OAC strategies in detail. Additionally, on the basis of our survey, no conclusions regarding safety and efficacy of the different NOAC approaches could be drawn because no data on the clinical outcomes of the patients were collected.

\section{Conclusions}

This nationwide survey provides the current practice and a 1-year trend of periprocedural OAC management of patients undergoing AF ablation at certified EP centers in Germany. While uninterrupted use of VKA has already been integrated in daily practice, there is a relevant variability in periprocedural NOAC strategies, whereby most EP centers discontinue NOAC therapy on the morning of the procedure (the minimally interrupted strategy). Future larger randomized clinical trials are warranted to compare the safety and efficacy of the more commonly practiced minimally interrupted periprocedural NOAC regimes with the truly uninterrupted periprocedural NOAC regimes. 


\section{Statement of Ethics}

The survey was conducted with the approval and support of the National Working Group on Arrhythmias of the German Cardiac Society (DGK).

\section{Conflict of Interest Statement}

C.M. has received speaking honoraria from Abbott, Bayer, Bristol-Myers Squibb, Boehringer Ingelheim, Boston Scientific, Daichii Sankyo, and consultant honorarium from Boston scientific and Biosense Webster/Johnson and Johnson. C.V. has received speaker honoraria from Abbott, Bayer, Boston Scientific, Biotronik, BMS, Boehringer Ingelheim, Daiichi Sankyo, Medtronic, Zoll. D.D. received lecture honoraria from Abbott, Astra Zeneca, Pfizer, Zoll. D.G.D. received lecture honoraria, consulting fees or travel expenses from Abbott, Bayer, Biotronik and Pfizer. D.L. received lecture honorarium from Microport, Medtronic, ResMed, Sanofi, Bayer. G.F. has received lecture honoraria, consulting fees or travel expenses from Abbott, Bayer, Biotronik, Daichii Sankyo, Medtronic and Pfizer. L.E. discloses consultant fees, speaking honoraria, and travel expenses from Abbott, Bayer Healthcare, Biosense Webster, Biotronik, Boehringer, Boston Scientific, Bristol-Myers Squibb, Daiichi Sankyo, Medtronic, Pfizer, and Sanofi.Aventis. Research has been supported by German Research Foundation (DFG) and German Heart Foundation outside the submitted work. P.M. has received speaking honoraria and travel expenses from Daichii Sankyo. R.W. has received speaking honoraria, consultant fees or travel expenses from Biotronik, Boston Scientific, Medtronic, Bayer, Daichii Sankyo, Bristol-Myers Squibb/Pfizer, Boehringer Ingelheim, Novartis; investigator-initiated funding for research projects (initiated by him) from BristolMyers Squibb/ Pfizer, and Boston Scientific.

\section{References}

1 Kirchhof P, Benussi S, Kotecha D, Ahlsson A, Atar D, Casadei B, et al.; ESC Scientific Document Group. 2016 ESC Guidelines for the management of atrial fibrillation developed in collaboration with EACTS. Eur Heart J. 2016 Oct;37(38):2893-962.

2 Santangeli P, Di Biase L, Horton R, Burkhardt JD, Sanchez J, Al-Ahmad A, et al. Ablation of atrial fibrillation under therapeutic warfarin reduces periprocedural complications: evidence from a meta-analysis. Circ Arrhythm Electrophysiol. 2012 Apr;5(2):302-11.

3 Di Biase L, Burkhardt JD, Santangeli P, Mohanty P, Sanchez JE, Horton R, et al. Periprocedural stroke and bleeding complications in patients undergoing catheter ablation of atrial fibrillation with different anticoagulation management: results from the Role of Coumadin in Preventing Thromboembolism in Atrial Fibrillation (AF) Patients Undergoing Catheter Ablation (COMPARE) randomized trial. Circulation. 2014 Jun;129(25):2638-44.

4 Cappato R, Marchlinski FE, Hohnloser SH, Naccarelli GV, Xiang J, Wilber DJ, et al.; VENTURE-AF Investigators. Uninterrupted rivaroxaban vs. uninterrupted vitamin $\mathrm{K}$ antagonists for catheter ablation in non-valvular atrial fibrillation. Eur Heart J. 2015 Jul;36(28): 1805-11.

5 Calkins H, Willems S, Gerstenfeld EP, Verma A, Schilling R, Hohnloser SH, et al.; RE-CIRCUIT Investigators. Uninterrupted Dabigatran versus Warfarin for Ablation in Atrial Fibrillation. N Engl J Med. 2017 Apr;376(17): 1627-36.
6 Kirchhof P, Haeusler KG, Blank B, De Bono J, Callans D, Elvan A, et al. Apixaban in patients at risk of stroke undergoing atrial fibrillation ablation. Eur Heart J. 2018 Aug;39(32):294255.

7 Hohnloser SH, Camm J, Cappato R, Diener HC, Heidbüchel H, Mont L, et al. Uninterrupted edoxaban vs. vitamin $\mathrm{K}$ antagonists for ablation of atrial fibrillation: the ELIMINATE-AF trial. Eur Heart J. 2019 Sep;40(36): 3013-21.

8 Calkins H, Hindricks G, Cappato R, Kim YH, Saad EB, Aguinaga L, et al. 2017 HRS/EHRA/ ECAS/APHRS/SOLAECE expert consensus statement on catheter and surgical ablation of atrial fibrillation: executive summary. Europace. 2018 Jan;20(1):157-208.

9 Steffel J, Verhamme P, Potpara TS, Albaladejo P, Antz M, Desteghe L, et al.; ESC Scientific Document Group. The 2018 European Heart Rhythm Association Practical Guide on the use of non-vitamin K antagonist oral anticoagulants in patients with atrial fibrillation. Eur Heart J. 2018 Apr;39(16): 1330-93

10 Romero J, Cerrud-Rodriguez RC, Diaz JC, Michaud GF, Taveras J, Alviz I, et al. Uninterrupted direct oral anticoagulants vs. uninterrupted vitamin $\mathrm{K}$ antagonists during catheter ablation of non-valvular atrial fibrillation: a systematic review and meta-analysis of randomized controlled trials. Europace. 2018 Oct;20(10):1612-20.

11 Reynolds MR, Allison JS, Natale A, Weisberg IL, Ellenbogen KA, Richards M, et al. A Pro- spective Randomized Trial of Apixaban Dosing During Atrial Fibrillation Ablation: the AEIOU Trial. JACC Clin Electrophysiol. 2018 May;4(5):580-8.

12 Nogami A, Harada T, Sekiguchi Y, Otani R, Yoshida Y, Yoshida K, et al.; ABRIDGE-J (ABlation peRIoperative DabiGatran in use Envisioning in Japan) Investigators. Safety and efficacy of minimally interrupted dabigatran vs. uninterrupted warfarin therapy in adults undergoing atrial fibrillation catheter ablation: a randomized clinical trial. JAMA Netw Open. 2019 Apr;2(4):e191994.

13 Nakamura K, Naito S, Sasaki T, Take Y, Minami K, Kitagawa Y, et al. Uninterrupted vs. interrupted periprocedural direct oral anticoagulants for catheter ablation of atrial fibrillation: a prospective randomized single-centre study on post-ablation thrombo-embolic and haemorrhagic events. Europace. 2019 Feb; 21(2):259-67.

14 Nagao T, Suzuki H, Matsunaga S, Nishikawa Y, Harada K, Mamiya K, et al. Impact of periprocedural anticoagulation therapy on the incidence of silent stroke after atrial fibrillation ablation in patients receiving direct oral anticoagulants: uninterrupted vs. interrupted by one dose strategy. Europace. 2019 Apr;21(4): 590-7.

15 Hohnloser SH, Basic E, Hohmann C, Nabauer M. Effectiveness and Safety of Non-Vitamin K Oral Anticoagulants in Comparison to Phenprocoumon: Data from 61,000 Patients with Atrial Fibrillation. Thromb Haemost. 2018 Mar;118(3):526-38. 